\title{
Examiner's finger-mounted near- infrared spectroscopy is feasible to analyze cerebral and skeletal muscle oxygenation in conscious Chihuahuas
}

Keisuke Hiwatashi

Kimiaki Doi

Risuke Mizuno

Makoto Yokosuka 


\title{
Examiner's finger-mounted near-infrared spectroscopy is feasible to analyze cerebral and skeletal muscle oxygenation in conscious Chihuahuas
}

\author{
Keisuke Hiwatashi, ${ }^{a, b, \dagger}$ Kimiaki Doi, ${ }^{c, \dagger}$ Risuke Mizuno, ${ }^{d}$ and Makoto Yokosuka ${ }^{a, *}$ \\ ${ }^{a}$ Nippon Veterinary and Life Science University, Laboratory of Comparative Medicine, School of Veterinary Medicine, Musashino, Tokyo, Japan \\ ${ }^{\mathrm{b}}$ Animal Medical Center of Gotemba Inter, Gotemba, Shizuoka, Japan \\ 'Doi Pet Clinic, Fujieda, Shizuoka, Japan \\ ${ }^{\mathrm{d} T}$ Tsukuba International University, Department of Medical Technology, Faculty of Health Sciences, Tsuchiura, Ibaraki, Japan
}

\begin{abstract}
To measure regional saturation of oxygen $\left(\mathrm{rSO}_{2}\right)$ of hemoglobin and total hemoglobin index $(\mathrm{Hbl})$ in the brain (through the molera of the head) and skeletal muscle (musculus gracilis) of conscious Chihuahua dogs using an examiner's finger-mounted near-infrared spectroscopy (NIRS) device, Toccare, we investigated brain and skeletal muscle NIRS in 48 Chihuahuas without severe disease. To measure $\mathrm{rSO}_{2}$ and total $\mathrm{Hbl}$, a Toccare probe was placed on the molera of the head and musculus gracilis of each dog for real-time recording. Stable NIRS values were obtained within $10 \mathrm{~s}$. We also examined the effect of anesthesia on $\mathrm{rSO}_{2}$ and total $\mathrm{Hbl}$ of a Chihuahua. Cerebral $\mathrm{rSO}_{2}$ values $(59 \% \pm 7 \%)$ were significantly lower than those obtained at femoral regions $(67 \% \pm 6 \%)$, whereas total $\mathrm{Hbl}$ values in the brain $(0.38 \pm 0.09)$ were significantly higher than those of the musculus gracilis $(0.20 \pm 0.05)$. Sedation with a combination of medetomidine and ketamine decreased cerebral $\mathrm{rSO}_{2}$ along with a corresponding reduction in heart rate. Sevoflurane anesthesia with $100 \% \mathrm{O}_{2}$ maintained $\mathrm{rSO}_{2}$ in the brain with an even lower heart rate. In conclusions, we measured brain and skeletal muscle rSO $\mathrm{S}_{2}$ of hemoglobin in conscious Chihuahuas using a newly developed NIRS device, Toccare, and found that changes in cerebral oxygenation levels were associated with administration of anesthetics. () The Authors. Published by SPIE under a Creative Commons Attribution 3.0 Unported License. Distribution or reproduction of this work in whole or in part requires full attribution of the original publication, including its DOI. [DOI: 10.1117/1.JBO.22.2.026006]
\end{abstract}

Keywords: brain; microcirculation; hemoglobin; imaging.

Paper 160764R received Nov. 4, 2016; accepted for publication Jan. 26, 2017; published online Feb. 15, 2017.

\section{Introduction}

Near-infrared spectroscopy (NIRS) is a unique visualization technology used to measure tissue oxygen saturation of hemoglobin at the capillary level. ${ }^{1}$ Although pulse oximetry detects the oxygen saturation of hemoglobin in pulsatile arterial blood of the finger or ear, ${ }^{2}$ it cannot be used to analyze the oxygen saturation in nonpulsatile environments including low-pressure areas and the peripheral microcirculation system. NIRS is thus a clinically useful and advantageous tool for detecting oxygenation levels in local and microcirculatory spaces through the skin and bones and has been used as a noninvasive clinical method in human patients. ${ }^{3,4}$ Several investigators have conducted experimental NIRS studies of $\operatorname{skin}^{5}$ and skeletal muscle ${ }^{6-9}$ in dogs. There are a few reports to evaluate cerebral oxygenation level using canine brain NIRS ${ }^{10}$ in comparison with human medicine. ${ }^{1,3}$

Neuronal cells, astrocytes, pericytes, and capillary endothelial cells in the central nervous system constitute a neurovascular unit. ${ }^{11}$ Neuronal activity and metabolism are closely related to regulation of the cerebral microcirculation, indicating

\footnotetext{
*Address all correspondence to: Makoto Yokosuka, E-mail: mayokosuka@nvlu .ac.jp
}

†These authors equally contributed to this study. that brain autoregulation mechanisms play significant roles in maintaining vital functions of the central nervous system. ${ }^{12}$ Recently, Kanayama and Niwayama ${ }^{13}$ developed an extremely small and mobile NIRS (Toccare, an examiner's finger-mounted type) that is suitable for human fetuses and neonates, and they were able to noninvasively measure fetal cerebral tissue oxygenation levels. ${ }^{14}$ It is very important to determine local brain oxygen saturation of hemoglobin in a conscious condition because sedation and anesthesia decisively affect cerebral circulation and neuronal activity/metabolism. ${ }^{15}$

Among companion dogs, most Chihuahuas have a molera of the head, and the lacuna may remain open throughout life. Therefore, we realized that a Chihuahua molera is a boneless locus and, consequently, NIRS beams easily reach the cerebral cortex by passing through the molera in the scalp, and is, therefore, ideal for a pilot study of canine brain NIRS in conscious state. In this study, we attempted to measure brain oxygenation levels in conscious Chihuahuas by applying a Toccare probe to the molera of the head. The objectives of this study were as follows: (1) to measure regional saturation of oxygen $\left(\mathrm{rSO}_{2}\right)$ and total hemoglobin index (HbI) in the cerebral region of conscious Chihuahuas using an examiner's finger-mounted NIRS (Toccare), (2) to compare results between brain and femoral NIRS, and (3) to evaluate the effects of anesthesia on cerebral and femoral NIRS values. 


\section{Materials and Methods}

\subsection{Animals}

Forty-eight Chihuahuas that were hospital outpatients at Animal Medical Center of Gotemba Inter in Gotemba City or Doi Pet Clinic in Fujieda City, Shizuoka Prefecture, Japan, were evaluated in this study. The dogs visited the clinics for regular health examinations and/or vaccinations. By veterinarian physical examinations, we confirmed that 48 dogs demonstrated no severe pathophysiological symptoms, although some (29\%) had mild symptoms including dermatitis, arthritis, gastroenteritis, blepharitis, or a slipped disk. They did not take any drugs or treatments before the NIRS measurements.

\subsection{NIRS Equipment}

The Toccare tissue oxygenation monitor (model KN-15) and its NIRS probe were used in this study. The measurement method of this device depends on spatially resolved NIRS with two LED light sources (770 and $830 \mathrm{~nm}) .{ }^{13}$ Toccare enabled us to measure $\mathrm{rSO}_{2}(0 \%$ to $99 \%)$ and total $\mathrm{HbI}(0$ to 1.0$)$ of each organ at $5-\mathrm{mm}$ depth with a sampling interval of $0.5 \mathrm{~s}$. Values of $\mathrm{rSO}_{2}$ and total $\mathrm{HbI}$ were directly displayed on the monitor of the Toccare and also recorded on a secure digital memory card. To perform NIRS, we did not need to prepare a dark room and shading component due to the high power of the Toccare LED.

\subsection{Experimental Protocol}

This study was performed following informed consent from owners and animal care approval from the Shizuoka Veterinary Medical Association. Figure 1 shows CT images (left panel, three-dimensional reconstruction; right panel, coronal plane) of the anatomical distribution and location of the molera in a Chihuahua under inhalation isoflurane anesthesia. The Toccare probe was applied to the molera of the head [Figs. 2(a) and 2(b)] and the left or right gracilis muscle. To tightly adhere the probe against the skin, we gently pushed hair aside using a hairbrush and wetted cotton. In the protocols for the conscious condition, dogs were sitting on the examination table during brain NIRS measurements and standing on the table during skeletal muscle NIRS. After attaching the NIRS probe, we could obtain a stable value of the brain and femoral NIRS within $10 \mathrm{~s}$ in conscious Chihuahuas.

To evaluate effects of anesthesia on the brain and femoral NIRS in a Chihuahua [Fig. 2(c)], inhalation of sevoflurane (1\%) with $100 \% \mathrm{O}_{2}$ was used for a minor surgery (castration) after sedation with a combination of medetomidine [30 $\mu \mathrm{g} / \mathrm{kg}$, im (intramuscular injection)] and ketamine $(2 \mathrm{mg} / \mathrm{kg}$, im). Mask anesthesia was used for the castration. After the surgery, we injected atipamezole $(75 \mu \mathrm{g} / \mathrm{kg}$, im) into a dog to antagonize the medetomidine-mediated sedation. We measured the NIRS values, heart rates (beats/min), and respiratory times (times/min) before and after anesthesia. In this protocol, we placed the dog in appropriate positions, such as standing, sitting, or prone, corresponding to sedative and anesthetic conditions.

\subsection{Statistical Analysis}

We evaluated the data for 48 Chihuahuas, and the resulting values are presented as the mean $\pm \mathrm{SD}$, with $n$ indicating the number of animals. Significant differences $(P<0.05)$ were determined using paired Student's $t$-test for comparisons between cerebral and femoral values or unpaired Student's $t$-test for comparisons between genders. A regression analysis was also performed using KaleidaGraph version 4.5.1 software.

\section{Results}

\subsection{Age, Gender, and Body Weight of Chihuahuas}

Chihuahuas in this study were between the ages of 0.3 and 16.5 years, with a mean age of $6.6 \pm 3.7$ years $(n=48)$. Fifteen dogs $(31 \%)$ were female, including some that were neutralized. The body weights of the dogs were between 1.6 and $6.0 \mathrm{~kg}$, with a mean body weight of $3.5 \pm 1.2 \mathrm{~kg}(n=48)$. There were no significant relationships between age and body weight of Chihuahuas (Fig. 3), indicating that there are neither linear nor sigmoidal distributions.
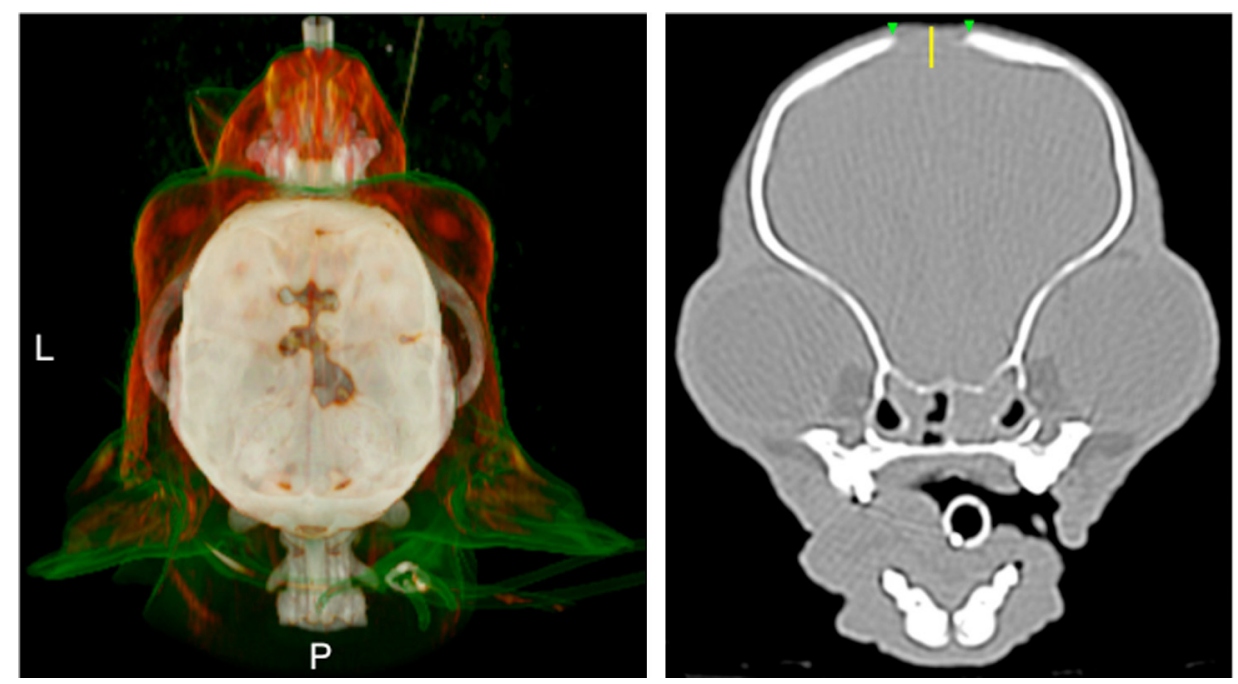

Fig. 1 Computed tomography images (left panel, three-dimensional reconstruction; right panel, coronal plane) of a Chihuahua under intratracheal isoflurane anesthesia with $100 \% \mathrm{O}_{2}$. A nonwhite area over the skull indicates the distribution of the molera (green arrowheads in right panel). $L$ and $P$ in left panel indicate left and posterior, respectively. The yellow line in the right panel represents a length of $5 \mathrm{~mm}$. 

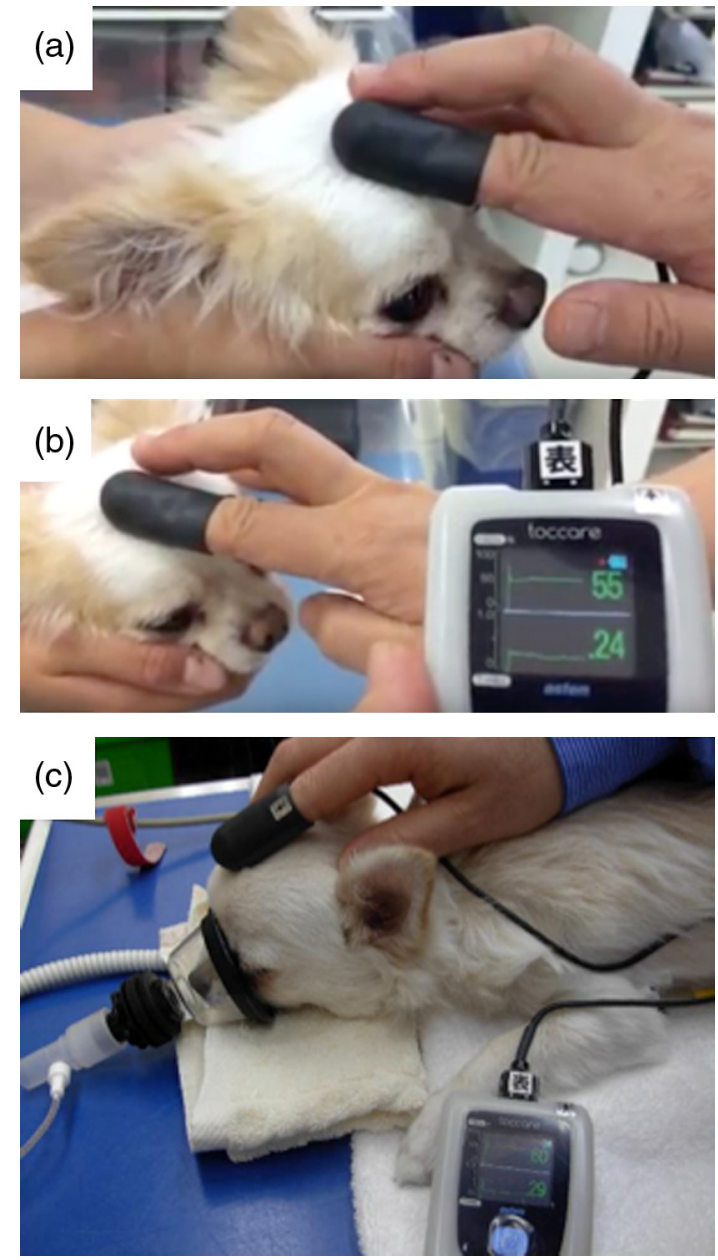

Fig. 2 A representative picture showing measurement of canine brain NIRS in (a) and (b) a conscious and (c) mask-anesthetized condition. Under a tranquil environment in a consulting room, a conscious Chihuahua was gently held by a veterinary nurse to prevent body movement during the NIRS measurement. A Toccare NIRS probe was placed on the molera of the head. The real-time values of $\mathrm{rSO}_{2}$ and total $\mathrm{Hbl}$ are displayed on the monitor of the Toccare (b) and (c).

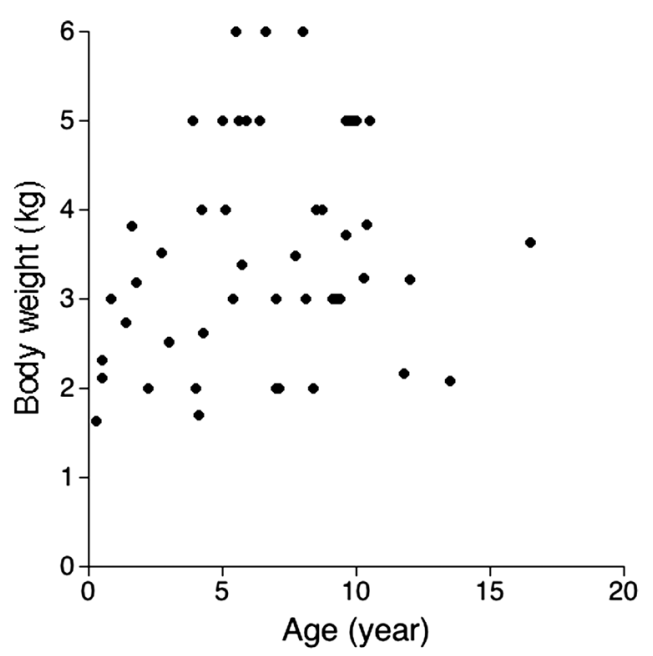

Fig. 3 Distribution of age and body weight of Chihuahuas in this study $(n=48)$.
Table 1 NIRS parameters $\left(\mathrm{rSO}_{2}\right.$ and total $\left.\mathrm{Hbl}\right)$ in cerebral and femoral parts of male and female dogs.

\begin{tabular}{lccccc} 
& \multicolumn{2}{c}{ Male $(n=33)$} & & \multicolumn{2}{c}{ Female $(n=15)$} \\
\cline { 2 - 3 } \cline { 6 - 6 } Parts & $\mathrm{rSO}_{2}(\%)$ & Total $\mathrm{Hbl}$ & & $\mathrm{rSO}_{2}(\%)$ & Total Hbl \\
\hline Brain & $58 \pm 7$ & $0.37 \pm 0.09$ & & $61 \pm 6$ & $0.40 \pm 0.09$ \\
Femoral & $67 \pm 5$ & $0.20 \pm 0.05$ & & $69 \pm 7$ & $0.20 \pm 0.05$ \\
\hline
\end{tabular}

\subsection{Values of $\mathrm{rSO}_{2}$ and Total $\mathrm{Hbl}$ in Cerebral and Femoral Regions}

The femoral $\mathrm{rSO}_{2}(67 \% \pm 6 \%)$ of the Chihuahuas was significantly higher than the cerebral $\mathrm{rSO}_{2}(59 \% \pm 7 \% ; P<0.05)$. The cerebral total $\mathrm{HbI}(0.38 \% \pm 0.09 \%)$ of the dogs was significantly higher than the femoral total $\mathrm{HbI}(0.20 \% \pm 0.05 \%$; $P<0.05)$. We also compared $\mathrm{rSO}_{2}$ and total $\mathrm{HbI}$ between male and female dogs and found no significant differences in those parameters (Table 1).

\subsection{Relationships of Age or Body Weight Against NIRS Values}

Figure 4 shows regression analyses for (a) and (b) age and (c) and (d) body weight (a) and (c) against $\mathrm{rSO}_{2}$ and (b) and (d) total $\mathrm{HbI}$ in the cerebral (red) and femoral (blue) regions in 48 Chihuahuas. There were slightly positive linear relationships between age and $\mathrm{rSO}_{2}$ in the femoral regions [Fig. 4(a), blue line; $y=64.333+0.46136 x, R=0.30097$ ], whereas the brain $\mathrm{rSO}_{2}$ remained almost the same regardless of age [Fig. 4(a), red line; $y=58.789-0.043461 x, R=0.022826]$. The relationships between age and total $\mathrm{HbI}$ in cerebral [Fig. 4(b), red line; $y=0.38471-0.00033282 x, R=0.01372]$ and femoral [Fig. 4(b), blue line; $y=0.20874-0.001348 x, R=0.1017$ ] regions were nonlinear as were the relationships between body weight and $\mathrm{rSO}_{2}$ in cerebral [Fig. 4(c), red line; $y=55.021+$ $0.98257 x, R=0.17224$ ] and femoral [Fig. 4(c), blue line; $y=$ $69.174-0.50232 x, R=0.10937]$ regions. There were slightly negative linear relationships between body weight and total $\mathrm{HbI}$ in the cerebral regions [Fig. 4(d), red line; $y=0.46654-$ $0.023736 x, R=0.32658$ ], whereas positive linear relationships were observed in the femoral regions [Fig. 4(d), blue line; $y=0.14893+0.014365 x, R=0.36173]$.

\subsection{Effects of Anesthesia on NIRS Values}

Finally, we examined the effects of inhalation anesthesia (sevoflurane) on changes in the $\mathrm{rSO}_{2}$ and total $\mathrm{HbI}$ of brain and femoral muscle during minor surgery of a Chihuahua (6-month-old male with a body weight of $2.14 \mathrm{~kg}$ ). Figure 5 shows the time course of changes in $\mathrm{rSO}_{2}$ (top panel) and total $\mathrm{HbI}$ (middle panel) of the cerebral (red symbols and lines) and femoral (blue symbols and lines) regions, and heart rates (bottom panel, closed circles) and respiratory times (bottom panel, open circles) in a dog before and after sedation and anesthesia. Excessive stress increased cerebral $\mathrm{rSO}_{2}$ (from $51 \%$ to $61 \%$ ) of the dog just before sedation with medetomidine and ketamine. After injection of the sedative, $\mathrm{rSO}_{2}$ values gradually decreased to reach $47 \%$. The changes in $\mathrm{rSO}_{2}$ before and after sedation corresponded with fluctuations in heart rate. During sevoflurane anesthesia with $100 \%$ oxygen, the heart rate was still low (after 

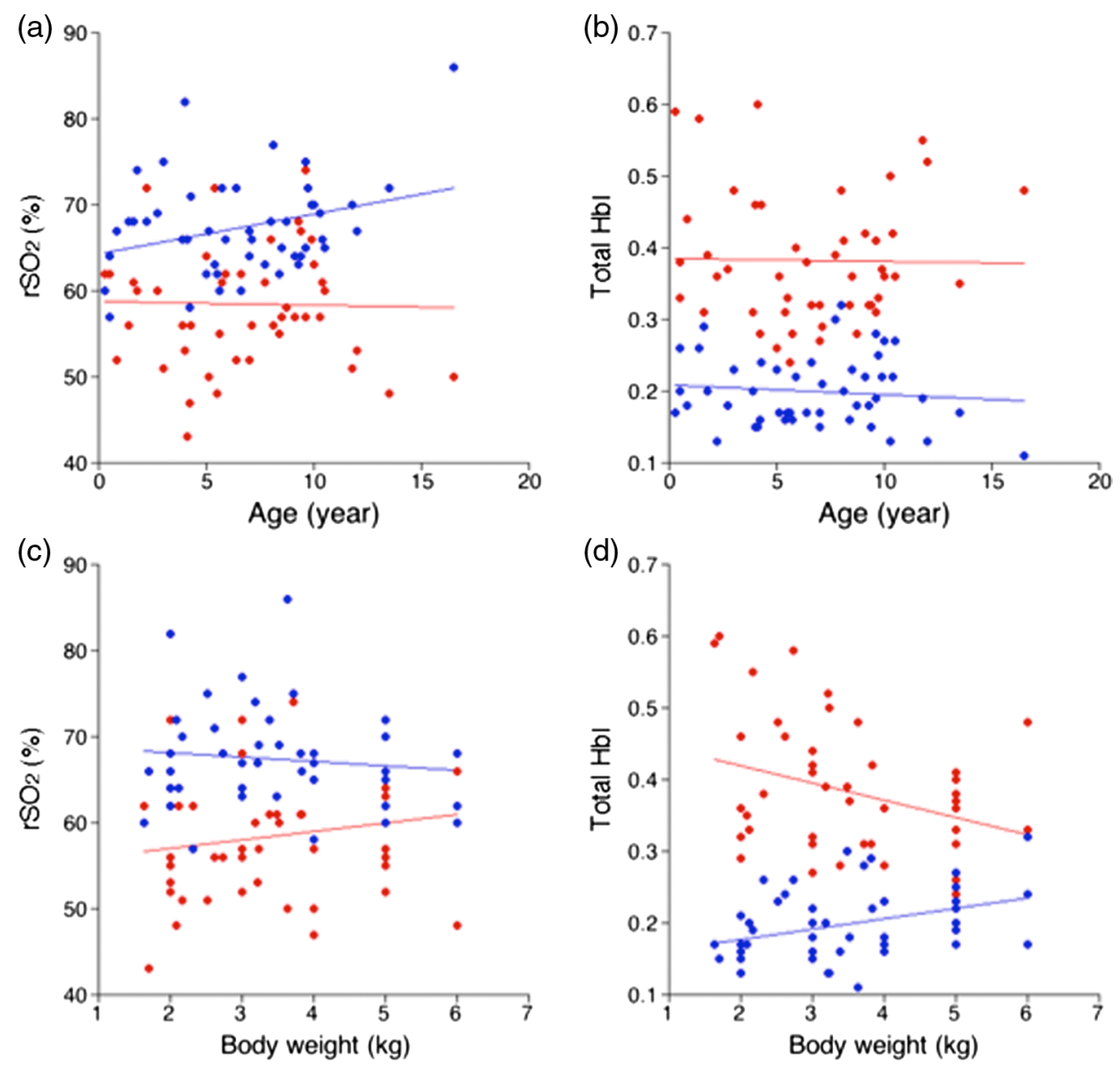

Fig. 4 Regression analyses for (a) and (b) age or (c) and (d) body weight (a) and (c) against $\mathrm{rSO}_{2}$ and (b) and (d) total $\mathrm{Hbl}$ at the cerebral (red) and femoral (blue) regions of 48 Chihuahuas.

sedation, 94 beats/min; during anesthesia, 82 beats/min), whereas $\mathrm{rSO}_{2}$ increased from $47 \%$ to $56 \%$. The cerebral $\mathrm{rSO}_{2}$ values were relatively stable (55\% to $60 \%)$ during anesthesia and recovery at $15 \mathrm{~min}$. A sudden increase in the femoral total $\mathrm{HbI}$, associated with shivering, was observed at the 0 min recovery time.

\section{Discussion}

In this study, we could measure cerebral oxygen saturation of hemoglobin in conscious Chihuahuas using a newly developed NIRS device, Toccare, for the first time. In the Toccare probe, the distance between the emitter and detectors was $8 \mathrm{~mm}$ and the width of the detectors was $3.5 \mathrm{~mm} .{ }^{13}$ Thus, to perform brain NIRS in Chihuahua, we needed at least an $8 \times 3.5 \mathrm{~mm}$ square as a measurement window. The Toccare LED beams reached a sub-tissue space of 5-mm depth (Fig. 1, right panel), indicating that we acquired oxy- and deoxyhemoglobin signals from the surface and superficial layers of the brain cortex. These optical characteristics indicate that the three-dimensional structure of the skull and the location of the molera are important determinants for accurate detection of signals during brain NIRS in dogs with a molera. Several dog breeds such as Maltese, Yorkshire terrier, English bulldog, lhasa apso, pug, and Pekingese possess a molera on the head, ${ }^{16}$ indicating that the Toccare can be used to measure and monitor brain oxygenation levels in other dogs in addition to Chihuahuas.
Originally, using Toccare, Uchida et al. measured cerebral $\mathrm{rSO}_{2}$ at the frontal region of the head of a human fetus ${ }^{14}$ because this region of the fetal head is hairless and comprises thin bones. The canine molera is usually located over the parietal region of the head (Fig. 1). In dogs, the top part of the brain is considered to be motor and somatosensory areas. ${ }^{17}$ The Brodmann areas have been well established in humans and other primates for understanding neuronal connectivity and brain functions. Unlike the classification of the feline brain, ${ }^{18}$ morphological and functional brain mappings in dogs remain unclear. Collectively, the noninvasive measurement of Chihuahua brain NIRS using Toccare may overcome the difficulty encountered in neuroscience and veterinary medicine regarding the evaluation of motor and somatosensory functions in a conscious state, although we recognize that it is necessary to improve NIRS so that it can detect cerebral $\mathrm{rSO}_{2}$ at increased depths. Recently, Uchida et al., ${ }^{19}$ demonstrated that an examiner's finger-mounted tissue oximetry, which is same device in this study, allowed measuring mice brain NIRS as an experimental animal model. Collectively, we propose here that using Toccare Chihuahua brain NIRS will intermediate between rodents and humans investigations.

Gygax et al. ${ }^{10}$ investigated NIRS of the brain functions of conscious beagles in response to a command. In this study, they demonstrated changes in the blood oxygenation level in the canine frontal brain area, and they did not elucidate the percentage of oxygen saturation of hemoglobin. In this study, we could detect $\mathrm{rSO}_{2}(\%)$ in brain as well as muscle of thigh in 
conscious Chihuahuas. The cerebral $\mathrm{rSO}_{2}$ values were significantly lesser than those of the skeletal muscles. We speculate that the discrepancy of $\mathrm{rSO}_{2}$ values between brain and hind leg in the conscious condition results were because (1) brain oxygen consumption would be maintained at a higher level compared with other parts of the body because neuronal activity and metabolism strictly govern the cerebral microcirculation through the neurovascular unit and autoregulation mechanisms, ${ }^{11,12}$ and (2) the oxygen demand of skeletal muscles would be less because the hind leg was in a resting condition. Through this study, we could exhibit obvious heterogeneity of oxygenation levels among organs in conscious dogs.

In a human study, fetal cerebral $\mathrm{rSO}_{2}$ measured using the same device was $65.5 \% \pm 8.58 \%,{ }^{14}$ which is slightly higher than that measured in canine brain and similar to the femoral values in this study. The discrepancy between human fetal cerebral $\mathrm{rSO}_{2}$ values and the current results in canine brain is quite intricate to explain because many factors including species differences and conditions, e.g., body position or consciousness, need to be taken into account. We understand that measuring area at the brain surface of Chihuahuas was anatomically different from human studies, ${ }^{14}$ and that the NIRS values obtained in this study are a part of molera and specific parameters for Chihuahuas. There were no significant linear relationships between age or body weight and brain $\mathrm{rSO}_{2}$, whereas the femoral $\mathrm{rSO}_{2}$ weakly depended on age in Chihuahuas [Figs. 4(a) and $4(\mathrm{c})]$. We also identified the similarity of cerebral $\mathrm{rSO}_{2}$ in male and female dogs (Table 1). These results suggest that irrespective of gender, age, or body weight, the cerebral oxygenation level of Chihuahuas is constantly maintained in the conscious condition when dogs are kept quiet in a restful environment; in other words, anxiety or excitement in dogs will generate unusual cerebral $\mathrm{rSO}_{2}$ patterns.

We also evaluated another NIRS parameter, total $\mathrm{HbI}$, in the brain and femoral regions. It is conceivable that the total $\mathrm{HbI}$ value represents the summation of oxy- and deoxy-hemoglobin per unit area. As the hemoglobin content in red blood cells is unchanged, the total hemoglobin may estimate the tentative blood flow within the local and microcirculatory basin containing arterioles, capillaries, and venules. The total $\mathrm{HbI}$ values in cerebral regions were significantly higher than those obtained at the femoral skeletal muscles in this study, suggesting that in the conscious state, brain blood flow per unit area may be greater than it is in the hind legs. Interestingly, in Fig. 4(d), the slopes of body weight versus total $\mathrm{HbI}$ of the brain and femoral regions were negative and positive, respectively, whereas both total $\mathrm{HbI}$ values were independent of age [Fig. 4(b)]. We thus assume that an increase in body weight may reduce cerebral blood flow in Chihuahuas, suggesting that obese subjects may be affected by disorders related to brain microcirculatory dysfunction, such as hypoxia or ischemia. Further investigation will be needed to identify the physiological meaning of total $\mathrm{HbI}$ in a variety of organs in the future.

Finally, we evaluated the effects of anesthesia on $\mathrm{rSO}_{2}$ and total $\mathrm{HbI}$ of cerebral and femoral regions in Chihuahuas (Fig. 5). Using the NIRS protocol in series, we confirmed that Toccare could detect changes in the NIRS parameters in response to sedation and anesthesia. Cerebral oxygenation levels were well maintained under sevoflurane inhalation with $100 \% \mathrm{O}_{2}$ even though the anesthetized dog exhibited a low heart rate. Although we could detect changes in the parameters of brain NIRS with $100 \% \mathrm{O}_{2}$ inhalation, the values were not statistically

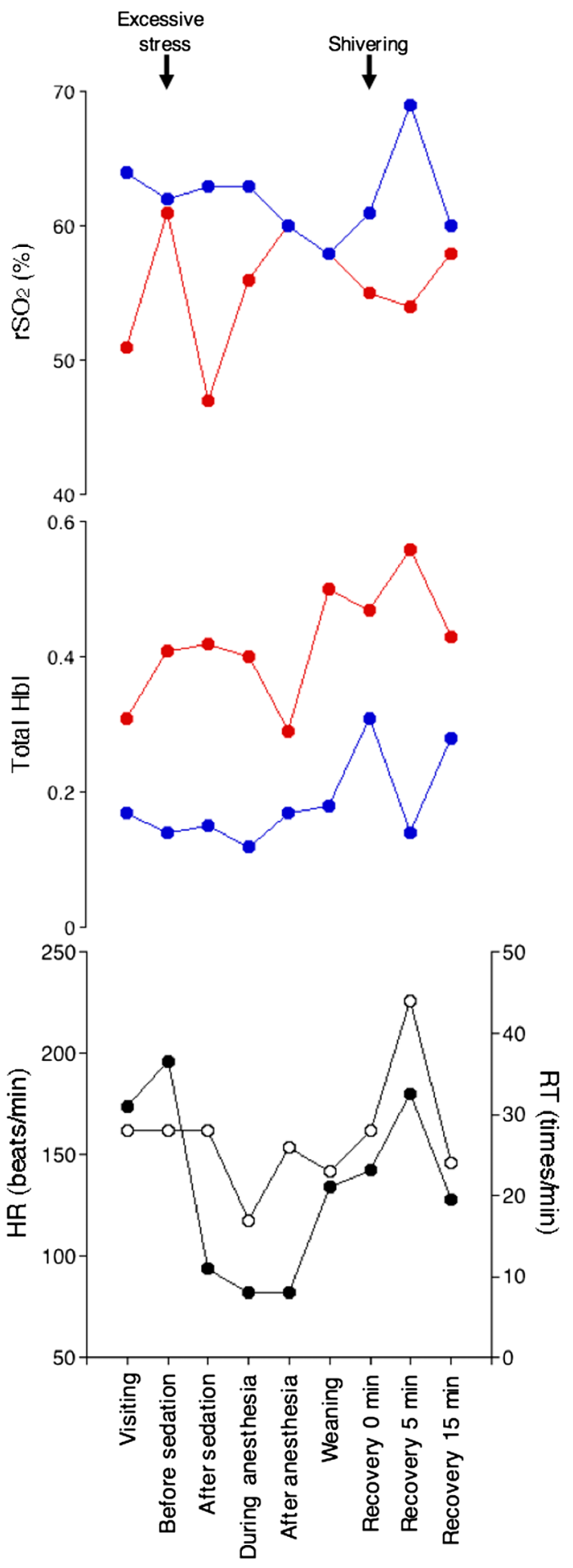

Fig. 5 Time-dependent changes in $\mathrm{rSO}_{2}$ (top panel) and total $\mathrm{Hbl}$ (middle panel) of cerebral (red symbols and lines) and femoral (blue symbols and lines) regions of a Chihuahua before and after sedation/anesthesia. The bottom panel shows the heart rate (HR; closed circles) and respiratory times (RT; open circles) during the same time course. Arrows indicate canine behaviors.

analyzed due to only one dog data, indicating that further investigation will be needed to identify changes in the brain NIRS values in response to $\mathrm{O}_{2}$ inhalation and anesthesia. In human medicine, NIRS is beginning to be used to monitor cerebral oxygenation levels under general anesthesia. ${ }^{20}$ These results suggest 
that, in the future, NIRS may be advantageous for monitoring cerebral oxygenation in the brains of anesthetized dogs. Other than anesthesia, we anticipate Toccare can be used as an oxygenation monitoring device in a variety of situations including ischemia and reperfusion of organs, e.g., liver and kidney, during surgery. Given Toccare's mechanical characteristics including its size and weight, use of AAA batteries, mobility, ease of operation, and data storage capabilities, NIRS will enable us to measure and monitor $\mathrm{rSO}_{2}$ and total $\mathrm{HbI}$ in the open air during emergency and critical care. Rather than nuclear scanning for functional brain analysis, NIRS represents a clinically simple, safe, and noninvasive technique for detection of tissue and organ oxygenation levels in the microcirculation system.

\section{Conclusions}

The salient findings of this study are that we could measure oxygen saturation of hemoglobin in brain and skeletal muscle in conscious Chihuahuas using a newly developed NIRS, Toccare, and found that significant differences of $\mathrm{rSO}_{2}$ existed between organs and changes in brain oxygenation levels are associated with anesthesia. We believe that Toccare NIRS will be used as a noninvasive method for monitoring vital oxygenation levels, especially in brain, in medicine during surgery and emergency and critical care.

\section{Disclosures}

All authors declare that there are no financial or other relationships that might lead to conflict of interest.

\section{References}

1. J. M. Murkin and M. Arango, "Near-infrared spectroscopy as an index of brain and tissue oxygenation," Br. J. Anaesth. 103(Suppl 1), i3-i13 (2009).

2. A. Jubran, "Pulse oximetry," Crit. Care. 19, 272-278 (2015).

3. H. B. Nielsen, "Systematic review of near-infrared spectroscopy determined cerebral oxygenation during non-cardiac surgery," Front. Physiol. 5, 93 (2014).

4. R. P. E. Boezeman et al., "Systematic review of clinical applications of monitoring muscle tissue oxygenation with near-infrared spectroscopy in vascular disease," Microvasc. Res. 104, 11-22 (2016).

5. B. Cugmas et al., "Detection of canine skin and subcutaneous tumors by visible and near-infrared diffuse reflectance spectroscopy," J. Biomed. Opt. 20(3), 037003 (2016).

6. S. Engbers et al., "A comparison of tissue oxygen saturation measurements by 2 different near-infrared spectroscopy monitors in 21 healthy dogs," J. Vet. Emerg. Crit. Care. 24(5), 536-544 (2014).
7. N. D. Pavlisko et al., "Evaluation of tissue oxygen saturation with nearinfrared spectroscopy during experimental acute hemorrhagic shock and resuscitation in dogs," Am. J. Vet. Res. 75(1), 48-53 (2014).

8. N. D. Pavlisko et al., "Evaluation of tissue hemoglobin saturation $\left(\mathrm{StO}_{2}\right)$ using near-infrared spectroscopy during hypoxemia and hyperoxemia in beagle dogs," Vet. Anaesth. Analg. 43(1), 18-26 (2016).

9. K. E. Hall et al., "Measurement of tissue oxygen saturation levels using portable near-infrared spectroscopy in clinically healthy dogs," J. Vet. Emerg. Crit. Care. 18(6), 594-600 (2008).

10. L Gygax et al., "Dog behavior but not frontal brain reaction changes in repeated positive interactions with a human: a non-invasive pilot study using functional near-infrared spectroscopy (fNIRS)," Behav. Brain Res. 281, 172-176 (2015).

11. T. Dalkara and L. Alarcon-Martinez, "Cerebral microvascular pericytes and neurogliovascular signaling in health and disease," Brain Res. 1623, 3-17 (2015).

12. C.K. Willie et al., "Integrative regulation of human brain blood flow," J. Physiol. 592(5), 841-859 (2014).

13. N. Kanayama and M. Niwayama, "Examiner's finger-mounted fetal tissue oximetry," J. Biomed. Opt. 19(6), 067008 (2014).

14. T. Uchida et al., "Examiner's finger-mounted fetal tissue oximetry: a preliminary report on 30 cases," J. Perinat. Med. 44(7), 745-749 (2016).

15. V. Bonhomme et al., "Influence of anesthesia on cerebral blood flow, cerebral metabolic rate, and brain functional connectivity," Curr. Opin. Anaesthesiol. 24(5), 474-479 (2011).

16. B.A. Summers, J.O. Cummings, and A. De Lahuta, "Malformation of the central nervous system," in Veterinary Neuropathology, pp. 68-94, Mosby-Year Book, St. Louis (1995).

17. G. Rajkowska and A. Kosmal, "Contralateral connections of the dog's frontal association cortex," Acta. Neurobiol. Exp. 49(4), 141-151 (1989).

18. J.W. Scannell, C. Blakemore, and M.P. Young, "Analysis of connectivity in the cat cerebral cortex," J. Neurosci. 15(2), 1463-1483 (1995).

19. T. Uchida et al., "Craniofacial tissue oxygen saturation is associated with blood $\mathrm{pH}$ using an examiner's finger-mounted tissue oximetry in mice," J. Biomed. Opt. 21(4), 040502 (2016).

20. G. Hernandez-Meza et al., "Near-infrared spectroscopy for the evaluation of anesthetic depth," Biomed. Res. Int. 2015, 1-11 (2015).

Keisuke Hiwatashi graduated from Department of Veterinary Science, Nippon Veterinary and Animal Science University, Tokyo, Japan, in 1996. He received Japan national license of DVM from MAFF in 1996. From 1996-1998, he worked at the Animal Medical Center of Southern California, LA, USA. From 1998-1999, he worked at the Tazi Animal hospital, Japan. In 1999, he opened the Animal Medical Center of Gotemba Inter as the hospital director.

Kimiaki Doi graduated from Department of Veterinary Science, Nippon Veterinary and Animal Science University, Tokyo, Japan, in 1993. He received Japan national license of DVM from MAFF in 1993. From 1993-2004, he worked at the Morishita Animal hospital, Japan. In 2004, he opened the Doi Pet Clinic, Fujieda, Japan, as the hospital director.

Biographies for the other authors are not available. 